\title{
Observations on the microstructure of response conflict
}

\author{
JAMES D. ST. JAMES \\ University of Illinois at Urbana-Champaign, Champaign, Illinois
}

\begin{abstract}
Three experiments are detailed which indicate the usefulness of the frequency of double responses (DRs) as an indicant of response conflict, in addition to the usual RT measures. DRs are responses on which an initial (often partial) error response is made that is rapidly amended to a correct response. Such responses occur most frequently in stimulus conditions where response conflict is expected by theory to be present and is indicated by changes in RT. Partial activation of competing responses has been demonstrated before using physiological measures (Coles, Gratton, Bashore, C. W. Eriksen, \& Donchin, 1985; C. W. Eriksen, Coles, Morris, \& O'Hara, 1985) when both responses could physically be made at the same time. DRs seem to be an equivalent type of movement when the subject cannot physically make but one movement at a time. The usefulness of this index of response conflict lies in its ease of implementation.
\end{abstract}

I wish to make the point in this paper that the probability of a certain peculiarity of movement (a double response, henceforth DR) changes with changes in the type of stimulus presented, in a common reaction time (RT) paradigm. Specifically, DRs are more frequent in those situations where response conflict has been invoked as an explanation for RT differences. DRs occur when a subject begins a movement of a response lever in the incorrect direction and then makes a very rapid change to a movement in the correct direction. Detection of DRs requires going beyond simple latency measures of RT to examine the execution of the responses-their "microstructure."

Research by Coles, Gratton, Bashore, C. W. Eriksen, and Donchin (1985) has shown the usefulness of continuous recordings of pressure during a hand-squeeze response and EMG responses of muscles making those squeezes. In their research, the overt response was a handle-squeeze by the left or right hand, so both responses could be emitted on a single trial, which was found sometimes to occur. Target letters were displayed in a known location, with noise letters also in the display. When the noise types (compatible versus incompatible noise) occurred randomly within blocks of trials, squeeze activity on the incorrect side was more than twice as likely to occur for incompatible-noise trials than for compatible-noise trials. When this incorrect-side squeeze occurred, there was

This research was supported by Public Health Service Career Program Award K06-MH22014, Public Health Service Research Grant MH 01206, and a small grant from the University of Illinois Research Board to C. W. Eriksen, whose assistance in all phases of the research is greatfully acknowledged. I also wish to thank Richard Carlson for his help with both the programming and ideas. Requests for reprints should be addressed to the author at Millikin University, 1184 W. Main, Decatur, IL 62522. usually also a squeeze on the correct side, though with a longer latency.

C. W. Eriksen, Coles, Morris, and O'Hara (1985) have reported a similar response-conflict study, in which EMG activity was recorded during thumb-press responses with the two hands. In addition to the slower responses for incompatible noise, they found EMG activity in the muscles associated with the incorrect response on $40 \%$ of those trials, versus only $8 \%$ of trials with compatible noise. The incorrect EMG activity occurred prior to correct activity, as is noted in Coles et al. (1985).

The finding of response activation in a nonresponding hand has been reported before. Kantowitz (1973) proposed a response-conflict model of the refractory period in "double stimulation," and he tested this model by comparing the response force of buttonpresses under various conditions. Response force on the transducer operated by the hand that did not respond varied with interstimulus interval, suggesting the presence of a degree of response tendency when response conflict would be expected by theory to be present.

The aim in this paper is to show that an even simpler response recording can be used as an index of response conflict. A DR occurs when, following presentation of a stimulus, a subject initiates an overt movement of a response lever in the incorrect direction, but that initial error movement is quickly replaced by a movement in the opposite (correct) direction. DRs appear to be equivalent to the types of responses recorded by Coles et al. (1985) and C. W. Eriksen et al. (1985), except that the responses are both made with the same hand, and only a single recording (of response-lever position or simple switch closure) is required.

DRs have been studied before in the context of visual-manual step-tracking (e.g., Gibbs, 1965; Megaw, 1972), but that research has not directly addressed whether the probability of a DR might vary for different stimuli. 
Still, Gibbs (1965) and Megaw (1972) have shown that DRs are more frequent with incompatible response mapping (target moves left, move the lever right) than with compatible mapping, and Higgins and Angel (1970) have used reversals of stimulus-response mapping to produce DRs for studies suggesting that the "error corrections" result from a central (rather than muscular) feedback mechanism.

The three experiments reported here replicate the response-conflict paradigm of B. A. Eriksen and C. W. Eriksen (1974), with the addition of continuous recording of the position of the single response lever and manipulation of the criterion for a movement to count as a response. Continuous recordings of joystick responses during trials of a two-choice RT task were made, with joystick movements to the left and right as indicants of which of two targets (a letter $\mathrm{S}$ or $\mathrm{H}$ ) appeared above a central fixation marker. The targets were presented either alone (no noise-S or $\mathrm{H}$ ) or with flanking letters. When flanking letters were present, they were repetitions of the target (compatible noise-SSS or $\mathrm{HHH}$ ), or the letters $\mathrm{P}$ and $Q$, which have no defined response (neutral noisePSP, QSQ, PHP, or QHQ), or the opposite target (incompatible noise-HSH or SHS).

\section{EXPERIMENT 1}

\section{Method}

Subjects. Three male and 4 female students at the University of Illinois at Urbana-Champaign served as paid subjects. All had normal or corrected vision and were right-handed, by self-report.

Apparatus and Procedures. Stimulus presentation and data collection were controlled by a DEC LSI-11 microcomputer, using a Hazeltine 1500 CRT terminal. Viewing of the stimuli was binocular, from a distance of 36 in. The subjects placed their faces against a viewing hood to control the distance from the CRT screen. At the distance used, the characters presented as stimuli were $0.25^{\circ}$ of visual angle in height, with a center-to-center width of $0.16^{\circ}$. A fixation point $(+)$ was displayed $0.44^{\circ}$ below the target position. The fixation remained on display between trials and during stimulus presentation. The subject initiated stimulus presentation by pressing a footpedal. The stimulus, which was presented $500 \mathrm{msec}$ later, remained on for $200 \mathrm{msec}$. Stimulus presentation occurred in a darkened room. The stimuli were thus clearly visible, and in a known location.

The subject indicated a response by pressing a joystick to the right or left. The joystick used was a fingertip-controlled Measurement Systems Model 521R, with high-resolution potentiometers. The subjects were instructed to grip the joystick between the thumb and middle fingers, with the index finger resting on top. It was emphasized to them that they were to be gripping the joystick in that manner before initiating a trial, to ensure that the entire reaction was recorded.

Return-to-center detente springs centered the joystick in both axes, with a breakout force of $85 \mathrm{~g}$ and a pull of $170 \mathrm{~g}$ at the maximum deflection of $27.5^{\circ}$. If the joystick was not vertical at the time the subject initiated the trial, an error message was displayed. Vertical was defined as a region no more than $0.8^{\circ}$ from true vertical. An analog-to-digital converter permitted the collection of the positions of the joystick in units of $0.159^{\circ}$. Sampling was done every $10 \mathrm{msec}$, beginning with the subject's initiation of the trial, and continuing for $1,200 \mathrm{msec}$.
An initial series of 96 practice trials was given, followed by 320 test trials. The subjects completed the single session in about $1 \mathrm{~h}$. The targets ( $\mathrm{S}$ and $\mathrm{H}$ ) as well as the trial types occurred equally often, in random order. The subjects moved the joystick to the right for one target and to the left for the other, with direction of the correct reaction counterbalanced across subjects. The trial types were no noise (target letter only), compatible noise (target letter flanked by repetitions of itself), neutral noise (target letter flanked by Ps or Qs), and incompatible noise (target letter flanked by the opposite target letter). Only capital letters were used.

When flanking letters appeared, they were in locations immediately adjacent to the target. The width of the display (from the middle of the left flanking letter to the middle of the right flanking letter) was thus $.32^{\circ}$ of visual angle. The subjects were instructed to respond only to the middle letter, which always occurred above the fixation mark. The stimulus was displayed $500 \mathrm{msec}$ after the subject pressed the footswitch to begin each trial. The criterion deflection for a response was $7.97^{\circ}$.

Following the leverpress reaction, the CRT displayed both the RT and whether the reaction was correct or incorrect. The RT was the time from stimulus onset to the criterion deflection (correct or error) to the left or right, with an error reported if the deflection was in the wrong direction. The subjects were instructed to react to the target as quickly as possible, while keeping errors to a minimum, and they were warned verbally by the experimenter if the error rates were above $10 \%$.

DRs were not reported to the subjects. When DRs occurred, the trial was reported as an error if the initial deflection in the incorrect direction exceeded the criterion. If the initial error movement did not exceed the criterion, the trial was reported as correct.

\section{Results}

The results reported below (for all experiments) are based on univariate repeated measures analysis of variance (ANOVA), corrected by the Huyhn-Feldt procedure, with $\alpha=0.05$. Post hoc pairwise comparisons were performed with the Tukey honestly significant difference $(H S D)$ procedure.

Although the subjects were required to have the joystick positioned within close tolerances of vertical at the beginning of a trial, small constant errors could occur as a result of the subject's "leaning" slightly on the joystick. In order to remove this constant error, individual trials were corrected in the following manner: The mean joystick position was calculated for the time between the initiation of a trial and the stimulus onset, and this constant error was then subtracted from the measure of each of the subsequent samples of joystick position. This procedure also served to correct any error in calibration of the joystick.

With this correction, and on the basis of visual inspection of several hundred individual trials, a deflection of $1.59^{\circ}$ ( 10 "units") was chosen as indicating a voluntary movement by the subject. This deflection was small enough to detect nearly all movements (such as the initial error deflection in DRs), while large enough to rule out small, random fluctuations in voltage.

Reaction time. Mean correct RTs are reported in Table 1 . The analysis of these data was a repeated measures ANOVA, in which mean correct RT was compared for the four noise types, with trials on which DRs occurred excluded from the analysis. There was a significant differ- 
Table 1

Mean Correct Reaction Times, Percent Double Responses, and Clean Misses (with Standard Deviations), by Trial Type and Dependent Measure, Experiment 1

\begin{tabular}{|c|c|c|c|c|c|c|c|c|}
\hline \multirow{3}{*}{$\begin{array}{l}\text { Dependent } \\
\text { Measure }\end{array}$} & \multicolumn{8}{|c|}{ Noise Type } \\
\hline & \multicolumn{2}{|c|}{ No Noise } & \multicolumn{2}{|c|}{ Compatible } & \multicolumn{2}{|c|}{ Neutral } & \multicolumn{2}{|c|}{ Incompatible } \\
\hline & $M$ & $S D$ & $M$ & $S D$ & $M$ & $S D$ & $M$ & $S D$ \\
\hline Reaction time & 397 & 29 & 402 & 34 & 448 & 41 & 473 & 46 \\
\hline Double responses & 4.5 & 3.4 & 4.7 & 5.5 & 4.9 & 3.5 & 11.6 & 5.3 \\
\hline Clean misses (\%) & 3.0 & 2.2 & 2.5 & 2.2 & 5.6 & 3.1 & 18.1 & 13.2 \\
\hline
\end{tabular}

Note-Reaction times are in milliseconds and are given with double responses excluded.

ence between trial types $[F(3,18)=40.53]$. Pairwise comparisons indicated that the difference between no noise and compatible noise was not significant, and that neutral noise was significantly slower than no noise or compatible noise, with incompatible noise significantly slower than all other conditions ( $H S D=23 \mathrm{msec}$ ).

Double responses. Previous research (Coles et al., 1985) has shown that incorrect responses are sometimes initiated, even if the correct response is also emitted. In the present research, unlike in the Coles et al. experiment, a leverpress to the left or right was the response. Thus, subjects could not make both responses simultaneously. It is possible, however, that an incorrect response could be started and then amended to a correct response, these constituting together a DR. Note that when a DR occurred, it was scored as an error if it exceeded some arbitrary criterion deflection. Thus the number of DRs that were scored as errors was a function of the criterion deflection. For this reason, the comparison of the various stimulus conditions for the mean proportion of DRs includes both correct and error trials.

The overall test for mean differences in proportions of DRs was significant $[F(3,18)=9.18]$. Mean percent DRs for the four trial types are reported in Table 1. Pairwise comparisons revealed that significantly more DRs occurred in the incompatible-noise condition than in the other conditions $(H S D=4.2 \%)$. No other comparisons were significant.

Clean misses. Error trials on which no correct deflection occurred (there was no DR) will be termed clean misses. The mean percent of clean misses for the four trial types are also reported in Table 1 . The overall test for mean differences was significant $[F(3,18)=8.79]$. Pairwise comparisons revealed that there were significantly more clean misses in the incompatible-noise condition, with no other differences significant $(H S D=9.3 \%)$.

\section{Discussion}

It will be apparent to the reader that the general pattern of RTs was as expected, given previous research. RT was increased very slightly (though not significantly) by the introduction of repetitions of the target, but it was increased substantially by neutral noise, and still more strongly by incompatible noise.

One impetus for this research was the casual observation that DRs seemed to occur most often under condi- tions where response conflict was assumed (i.e., in the presence of incompatible stimuli), and a major focus for this experiment was to determine whether that was the case. DRs did indeed occur more often with incompatible noise than with any other noise type, with no other differences significant. The nature of responses themselves varies with stimulus conditions. That variation is not restricted solely to latency (RT). DRs only occur when priming of the incorrect response proceeds to the level of overt response activation. If priming of an incorrect response does not reach the level of overt movement, it may still be reflected in increased RT (and in muscle activation short of overt movement, as shown by Coles et al., 1985, and C. W. Eriksen et al., 1985).

Another point of note regarding the pattern of DR rates is that although DRs are considerably more frequent when incompatible noise is present, they are not more frequent for neutral noise. That is, there is no direct correspondence between patterns of RTs and DR rates. One possible interpretation of this finding is that the increase in RT with neutral noise is due not to response conflict, but rather to increased time for stimulus evaluation.

There was also a clear effect of the incompatible-noise condition on clean misses. This, along with the similar pattern of DRs, suggests that perhaps evidence for the opposite target, provided by the incompatible noise, sometimes builds up to the point of triggering a response. On some trials (DRs), the correct response is also begun, quickly enough to "correct" the initial error movement, whereas on other trials (clean misses) the correct movement either is not initiated, or is begun after the initial incorrect response has passed some "point of no return" (DeJong, Coles, Logan, \& Gratton, 1990).

\section{EXPERIMENT 2 Replication with Increased Power}

Experiment 2 replicated Experiment 1 in all details except the following. First, the number of experimental trials per subject was doubled, to 640 , in order to provide a more powerful examination of errors and double responses. Second, two groups of 8 subjects each were employed, which differed only in the criterion deflection for a response. One group had a very narrow criterion, which resulted in even a slight deflection of the joystick's being scored as a response, whereas the other group had 
a wide criterion, requiring a large deflection. The rationale behind these changes was to have one group (with the wide criterion) for whom double responses could occur reasonably frequently that did not count as errors because they did not exceed the criterion. This group would presumably not be attempting to suppress the double responses as much as the other group, for whom the criterion for a response was the same as the degree of deflection required to define a double response, when this (error) deflection was followed shortly by a movement in the correct direction. Thus, for the narrow-criterion group, all double responses were scored (and reported to the subject) as errors. Given the instruction to keep errors to a minimum, this was expected to encourage subjects to try to suppress double responses.

\section{Method}

Subjects. The wide-criterion group consisted of 2 males and 6 females; the narrow-criterion group consisted of 1 male and $7 \mathrm{fe}$ males. Two subjects were dismissed from the experiment due to high error rates during the practice trials.

Procedure. Stimulus displays and randomization were the same as for Experiment 1, except that the interval between the subject's footpress to initiate a trial and the onset of the stimulus was reduced to $200 \mathrm{msec}$. The subjects were tested in a single session, consisting of a block of 96 practice trials, followed by two blocks of 320 experimental trials each. A 10 -min break was required between the two experimental blocks.

Instructions to all subjects included an explanation of the procedure, instructions on how to hold the joystick, and an admonition to respond quickly but accurately. The instructions differed only in that the narrow-criterion group was told that "only a very small movement" of the joystick would count as a response, whereas the wide-criterion group was told that they would have to move the joystick "about two thirds of the way over" to its maximum deflection.

For the narrow-criterion group, the criterion deflection for a response was $1.59^{\circ}$, whereas for the wide-criterion group, it was $19.13^{\circ}$. RT, taken as the time from stimulus onset to the time the criterion deflection was reached, was displayed after each trial. Because small errors in calibration of the joystick could lead to false reports of errors for the narrow-criterion group, the average position of the joystick during the $200 \mathrm{msec}$ prior to stimulus onset was calculated, and this constant error was subtracted from the joystick position during the movement. This set the beginning position of the joystick at $0.00^{\circ}$, correcting for any errors of calibration. These corrected values of joystick position were used to determine the RT reported to the subject after each trial. (The criterion used in Experiment 1 was wide enough that minor errors in calibration of the joystick would not lead to false reports of errors, so this procedure was not used during the experiment. As noted above, it was used for later data analyses, and those analyses reflect the correction for calibration.)

\section{Results}

Initiation time. Reaction times for the four noise conditions are reported separately for the wide and narrow criteria in Table 2 . The criterion for a response was a confounding factor in the RT scores, since the wide-criterion group had farther to move than the narrow-criterion group. RT can be thought of as made up of initiation time (IT) to begin the movement, plus the time required to move to the criterion. Since the narrow criterion had been chosen to detect any movement greater than the noise level of the joystick and transmission lines, movement time for this condition is as close to zero as the instrumentation would reliably permit. Thus, RT for this condition is the same as IT. In order to avoid the confound of different movement times for the two criteria, in the analyses reported here IT (taken as time from stimulus onset to a deflection of $1.59^{\circ}$ ) was used for both groups (of course IT and RT are the same for the narrow criterion). Though not reported, the RTs for the wide criterion group averaged $47 \mathrm{msec}$ longer than the ITs, reflecting the time required to move the added distance. This time was nearly constant for the four trial types, ranging between 44 and $51 \mathrm{msec}$.

An analysis of IT (DRs excluded) was performed, with criterion as a between-groups factor, and noise type as a repeated measures factor. The between-groups main effect of criterion approached but did not reach significance $[F(1,14)=3.87]$, despite the sizable absolute difference in initiation times. The repeated measures main effect of noise type was significant $[F(3,42)=112.86]$. Pairwise comparisons revealed that all comparisons were significant $(H S D=14)$. The mean initiation times, averaged across criteria, were $418,435,469$, and $507 \mathrm{msec}$ for the no-noise, compatible-noise, neutral-noise, and incompatible-noise conditions, respectively. The interaction of criterion and noise type was also significant $[F(3,42)=4.26]$.

Follow-up repeated measures ANOVAs were performed to compare the noise conditions separately for the two criteria. Both analyses indicated significant differences $[F(3,21)=55.07$ and 60.38 for the wide and narrow criteria, respectively]. For both wide and narrow criteria, pairwise comparisons revealed significant differences for all comparisons except no noise versus compatible noise ( $H S D=16$ and 22 , respectively).

Double responses. The mean percent of DRs by noise condition and criterion deflection are presented in Table 3. The between-groups main effect of criterion deflection

Table 2

Means and Standard Deviations of Initiation Time (in milliseconds) by Trial Type and Criterion, Experiment 2

\begin{tabular}{|c|c|c|c|c|c|c|c|c|}
\hline \multirow[b]{3}{*}{ Criterion } & \multicolumn{8}{|c|}{ Noise Type } \\
\hline & \multicolumn{2}{|c|}{ No Noise } & \multicolumn{2}{|c|}{ Compatible } & \multicolumn{2}{|c|}{ Neutral } & \multicolumn{2}{|c|}{ Incompatible } \\
\hline & $M$ & $S D$ & $M$ & $S D$ & $M$ & $S D$ & $M$ & $S D$ \\
\hline Wide & 400 & 39 & 414 & 39 & 438 & 39 & 475 & 40 \\
\hline Narrow & 435 & 54 & 456 & 72 & 500 & 59 & 541 & 72 \\
\hline
\end{tabular}

Note-Double responses are excluded. 
Table 3

Means and Standard Deviations of Percent Double Responses by Trial Type and Criterion, Experiment 2

\begin{tabular}{|c|c|c|c|c|c|c|c|c|}
\hline \multirow[b]{3}{*}{ Criterion } & \multicolumn{8}{|c|}{ Noise Type } \\
\hline & \multicolumn{2}{|c|}{ No Noise } & \multicolumn{2}{|c|}{ Compatible } & \multicolumn{2}{|c|}{ Neutral } & \multicolumn{2}{|c|}{ Incompatible } \\
\hline & $M$ & $S D$ & $M$ & $S D$ & $M$ & $S D$ & $M$ & $S D$ \\
\hline Wide & 4.1 & 2.5 & 2.3 & 1.8 & 3.8 & 3.0 & 11.6 & 7.6 \\
\hline Narrow & 0.2 & 0.3 & 0.5 & 0.5 & 1.3 & 1.0 & 4.0 & 3.2 \\
\hline
\end{tabular}

Table 4

Means and Standard Deviations of Percent Clean Misses by Trial Type and Criterion, Experiment 2

\begin{tabular}{|c|c|c|c|c|c|c|c|c|}
\hline \multirow[b]{3}{*}{ Criterion } & \multicolumn{8}{|c|}{ Noise Type } \\
\hline & \multicolumn{2}{|c|}{ No Noise } & \multicolumn{2}{|c|}{ Compatible } & \multicolumn{2}{|c|}{ Neutral } & \multicolumn{2}{|c|}{ Incompatible } \\
\hline & $M$ & $S D$ & $M$ & $S D$ & $M$ & $S D$ & $M$ & $S D$ \\
\hline Wide & 1.5 & 2.2 & 1.6 & 1.2 & 1.9 & 1.5 & 6.4 & 5.4 \\
\hline Narrow & 1.0 & 1.1 & 2.3 & 2.6 & 2.7 & 2.3 & 7.3 & 4.1 \\
\hline
\end{tabular}

was significant $[F(1,14)=10.06]$, reflecting the larger percentage of DRs with the wide criterion $(5.5 \%)$ than with the narrow criterion $(1.5 \%)$. The repeated measures ANOVA testing the main effect of noise condition was also significant $[F(3,42)=21.28]$. The mean percent DRs for no noise, compatible noise, neutral noise, and incompatible noise were, respectively, $2.2 \%, 1.4 \%, 2.6 \%$, and $7.8 \%$. Pairwise comparisons indicate that there were significantly more DRs for the incompatible-noise condition, with no other comparisons significant $(H S D=2.4 \%)$. The interaction of criterion deflection and noise type was also significant $[F(3,42)=4.33]$. Follow-up ANOVAs on the wide and narrow criterion groups separately revealed significant effects of noise type for each analysis $[F(3,21)=13.53$ (wide criterion), and $F(1,21)=9.67$ (narrow criterion)]. For both analyses, Tukey's $H S D$ procedure reveals significantly more DRs for the incompatible noise than for any other condition, with no other comparisons significant $(H S D=4.55 \%$ and $2.2 \%$ for the wide and narrow margins, respectively). Additional analyses were done to compare the percentage of DRs for wide and narrow criterion groups separately for each noise type. These analyses showed that there were significantly more DRs for the wide criterion than for the narrow criterion only for incompatible noise $[F(1,7)=7.05]$. None of the other comparisons was significant $(F<1)$ in all cases.

Clean misses. Mean percent clean misses are presented in Table 4 by noise condition and criterion deflection. The between-groups comparison of wide and narrow criteria was not significant $[F(1,14)<1]$. The repeated measures analysis of the main effect of noise condition was significant $[F(3,42)=13.64]$. Pairwise comparisons indicated that there were significantly more clean misses in the incompatible-noise condition than in any other, with no other comparisons significant $(H S D=2.6 \%)$. The interaction of criterion and noise condition was not significant $(F<1)$.

\section{Discussion}

The general pattern of RT results is the same as in Experiment 1 , and it is in line with previous research. Again, the pattern of RTs is as expected, but it does not directly shed light on the questions posed in the research.

The results of the analyses of DRs are essentially the same as in Experiment 1. There is the additional finding that DRs were less frequent for the narrow criterion, but were not reduced to zero. Even when subjects were urged to avoid DRs by having all such trials reported to them as errors, they could not avoid such responses completely, especially with incompatible noise. A more forceful attempt to preclude DRs might be successful (though likely at the cost of increased RT), but subjects clearly do not "sit on their hands" under the instructions used.

It is clear that the clean miss rate was much lower (especially for the incompatible noise condition) in Experiment 2 than in Experiment 1. The very high clean miss rates for incompatible noise in that experiment were largely the result of 2 subjects who had exceptionally high error rates in that condition.

\section{EXPERIMENT 3 Effects of Noise Location}

Experiments 1 and 2 established a relationship between the nature of the flanking letters in the display and the degree of response conflict, as indexed by increases in $\mathrm{RT}$ and double response rates. If change in numbers of DRs is useful as an index of response conflict, it should be sensitive to experimental manipulations designed to reduce response conflict. B. A. Eriksen and C. W. Eriksen (1974) have shown that response conflict, as indexed by $\mathrm{RT}$, was reduced as incompatible flanking letters were moved to more peripheral positions in the display. Experiment 3 replicates the narrow-criterion condition of Experiment 2 , but with the additional within-groups factor of location of the flanking letters. On half of the trials, 
the flanking letters were contiguous with the target (as in the previous experiments); on the other half, the flanking letters were spaced farther to the left and right.

\section{Method}

Subjects. One male and 7 female subjects participated in this experiment.

Procedure. Procedures were the same as for the narrow-criterion group of Experiment 2, except for the location of the flanking letters. On half of the trials, the flanking letters were located $0.16^{\circ}$ to the right and left of the target, measured center-to-center; on the other half, the flanking letters were located $0.82^{\circ}$ to the right and left. These two types of flanking letters will be referred to as contiguous and spaced, respectively. Contiguous and spaced flanking letters occurred equally often, in random order throughout the trials of the experiment. The no-noise trials were randomly divided into contiguous and spaced categories to permit a complete experimental design.

\section{Results}

Reaction time. Table 5 reports the mean RTs by noise type and location of flanking letters. By definition, all DRs resulted in errors for Experiment 3, since the criterion for a response and the criterion for judging that a movement had been made were the same (i.e., RT and IT were equal). Hence, this analysis is restricted only to correct trials with no DR. Note also that RT for this criterion is equal to IT as used in Experiment 2.

The main effect of location was significant $[F(1,7)=$ 103.25], reflecting the generally faster RTs for spaced noise $(M=439 \mathrm{msec})$ compared to contiguous noise ( $M$ $=487 \mathrm{msec}$ ). The main effect of noise type was also significant $[F(3,21)=119.51]$. Pairwise comparisons revealed that the no-noise and compatible-noise conditions did not differ, while all other differences were significant $(H S D=16 \mathrm{msec})$. The interaction of noise type and location was also significant $[F(3,21)=68.02]$.

As a follow-up to the significant interaction, one-way repeated measures ANOVAs were performed separately for the contiguous and spaced noise locations. For the contiguous noise locations, the noise types differed significantly $[F(3,21)=114.92]$, while post hoc comparisons revealed that all pairs of means differed significantly except those for no noise and compatible noise $(H S D=$ $21 \mathrm{msec}$ ). Noise types also differed significantly for the spaced noise locations $[F(3,21)=7.90]$, with pairwise comparisons showing that the incompatible noise led to significantly longer RTs, with no other differences significant $(H S D=11 \mathrm{msec})$. The effect of neutral and incompatible noise was thus reduced for spaced noise locations, with the reduction nearing a zero effect for the neutral noise.

Double responses. Table 6 reports the mean percent DRs by noise type and location of the flanking letters. The main effect of location was significant $[F(1,7)=$ 17.71], reflecting the larger percentage of DRs for the contiguous noise $(0.8 \%)$ than for the spaced noise $(0.4 \%)$. The main effect of noise type was also significant $[F(3,21)$ $=5.15]$. Pairwise comparisons of the mean percentages of DRs (ignoring location) for no noise $(0.3 \%)$, compatible noise $(0.5 \%)$, neutral noise $(0.2 \%)$, and incompatible noise $(1.4 \%)$ failed to reveal any significant pairwise comparisons $(H S D=1.3 \%)$. This, however, is less interesting than the finding of a significant location $\times$ noise type interaction $[F(3,21)=5.11]$. Follow-up ANOVAs were performed for spaced and contiguous noise locations separately. No effect of noise type was found for the spaced noise $(F<1)$, but the effect of noise type was significant for the contiguous noise $[F(3,21)=11.08]$. Pairwise comparisons found a significant difference between the incompatible noise condition and all others, with no other comparisons significant $(H S D=1.3 \%)$.

Clean misses. The mean percent clean misses by noise type and location of flanking letters is reported in Table 7. The main effect of location was significant $[F(1,7)=$ 8.36], which reflects the greater number of clean misses

Table 5

Means and Standard Deviations of Reaction Times (in milliseconds) by Trial Type and Spacing, Experiment 3

\begin{tabular}{|c|c|c|c|c|c|c|c|c|}
\hline \multirow[b]{3}{*}{ Spacing } & \multicolumn{8}{|c|}{ Noise Type } \\
\hline & \multicolumn{2}{|c|}{ No Noise } & \multicolumn{2}{|c|}{ Compatible } & \multicolumn{2}{|c|}{ Neutral } & \multicolumn{2}{|c|}{ Incompatible } \\
\hline & $M$ & $S D$ & $M$ & $S D$ & $M$ & $S D$ & $M$ & $S D$ \\
\hline Contiguous & 435 & 55 & 449 & 55 & 504 & 60 & 558 & 71 \\
\hline Spaced & 435 & 52 & 433 & 54 & 436 & 51 & 450 & 53 \\
\hline
\end{tabular}

Note-Double responses are excluded. Reaction time $=$ initiation time.

Table 6

Means and Standard Deviations of Percent Double Responses by Trial Type and Spacing, Experiment 3

\begin{tabular}{|c|c|c|c|c|c|c|c|c|}
\hline \multirow[b]{3}{*}{ Spacing } & \multicolumn{8}{|c|}{ Noise Type } \\
\hline & \multicolumn{2}{|c|}{ No Noise } & \multicolumn{2}{|c|}{ Compatible } & \multicolumn{2}{|c|}{ Neutral } & \multicolumn{2}{|c|}{ Incompatible } \\
\hline & $M$ & $S D$ & $M$ & $S D$ & $M$ & $S D$ & $M$ & $S D$ \\
\hline Contiguous & 0.3 & 0.3 & 0.2 & 0.3 & 0.4 & 0.6 & 2.5 & 1.9 \\
\hline Spaced & 0.2 & 0.3 & 0.9 & 2.1 & 0.1 & 0.3 & 0.2 & 0.2 \\
\hline
\end{tabular}


Table 7

Means and Standard Deviations of Percent Clean Misses by Trial Type and Spacing, Experiment 3

\begin{tabular}{|c|c|c|c|c|c|c|c|c|}
\hline \multirow[b]{3}{*}{ Spacing } & \multicolumn{8}{|c|}{ Noise Type } \\
\hline & \multicolumn{2}{|c|}{ No Noise } & \multicolumn{2}{|c|}{ Compatible } & \multicolumn{2}{|c|}{ Neutral } & \multicolumn{2}{|c|}{ Incompatible } \\
\hline & $M$ & $S D$ & $M$ & $S D$ & $M$ & $S D$ & $M$ & $S D$ \\
\hline Contiguous & 0.7 & 0.7 & 0.5 & 0.7 & 1.6 & 1.2 & 6.8 & 6.8 \\
\hline Spaced & 0.7 & 0.8 & 0.4 & 0.6 & 0.4 & 1.0 & 0.5 & 0.7 \\
\hline
\end{tabular}

for the contiguous noise $(2.4 \%)$ than for the spaced noise $(0.5 \%)$. There was also a significant main effect of noise type $[F(3,21)=5.86]$, though the Tukey $H S D$ procedure revealed no significant pairwise comparisons. Mean percentages of clean misses by noise type were $0.7 \%, 0.5 \%$, $3.7 \%$, and $0.9 \%$ for no noise, compatible noise, neutral noise, and incompatible noise, respectively, with $H S D=$ $3.5 \%$.

Of more interest is the significant interaction between noise type and location $[F(3,21)=7.39]$. Follow-up ANOVAs showed no significant effect of noise type for the spaced noise letters $(F<1)$, but the effect was significant for the contiguous noise $[F(3,21)=6.79]$. Pairwise comparisons of the four noise types revealed that there were significantly more clean misses for the incompatible noise than for any of the other noise types, with no other comparisons significant $(H S D=4.5 \%)$.

\section{Discussion}

Manipulation of response conflict by noise type replicated the results of Experiments 1 and 2, and the manipulation of response conflict by location of flanking noise also produced the expected results. B. A. Eriksen and C. W. Eriksen (1974) showed that flanking noise that was moved away from the target letters produced smaller changes in RT with noise type than contiguous noise letters. That effect was replicated in this experiment. On the assumption that response conflict was reduced by making noise more peripheral, the predicted outcome of Experiment 3 was that the frequency of DRs would also be reduced by spacing the noise farther from the targets. This prediction was confirmed.

\section{GENERAL DISCUSSION}

The principal finding in these experiments was that the frequency of DRs is a function of the stimulus type. Movement execution-the response itself-changes with changes in the nature of the stimulus (and thus, presumably, with changes in the processing of the stimulus). This supports the conclusion of previous research (Coles et al., 1985; C. W. Eriksen et al., 1985) that examination of the microstructure of responses has important implications for research designed to determine the nature of information processing.

One potential benefit of using the frequency of DRs as an indicant of response conflict is the ease of recording such responses. Previous research cited above required sophisticated physiological measuring techniques that are not widely available to researchers. Although the research reported here was carried out with a computer equipped with an analog-to-digital converter to continuously record the position of a joystick during responses, two stop-clocks recording switch closures to the left and right for a simple lever would serve as well for detecting DRs. In this case, the criterion deflection would be set mechanically, but the result ought to be the same.

The finding of B. A. Eriksen and C. W. Eriksen (1974), that incompatible noise led to a much longer RT, was replicated in all three experiments, as was their finding that neutral noise led to a less pronounced slowing in RT. Their finding that these effects were lessened when the noise letters were moved farther from the target letter was also replicated in Experiment 3.

Across all the experiments, DRs were more frequent for incompatible noise than for any other stimulus condition. The only situation in which this was not true was the peripheral-noise condition of Experiment 3, where there was also no effect of stimulus type on RT. This is what would be expected if response conflict was reduced when noise was located peripherally, as B. A. Eriksen and C. W. Eriksen (1974) assumed.

Interestingly, although the number of DRs increased in parallel with the increase in RT for the incompatible noise, they did not parallel the smaller increase in RT for neutral noise. That is, neutral noise was not associated with an increase in DRs. Neutral noise that has little feature overlap with the target letters (as is the case here) would not be expected to cause priming of competing responses, though it might well require additional processing time before the stimulus array could be evaluated for target identity and target location. This would result in the pattern found-an increase in RT, but not DR rate, with neutral noise. This suggests that the increase in RT with neutral noise may reflect an increased time to evaluate the stimulus while not reflecting response conflict.

The use of frequency of DRs as an indicant of response conflict has been successfully applied to testing competing models in a different domain-the "fast-same" effect in same-different judgments. In contrast to models that postulate stimulus-evaluation mechanisms to account for this effect (e.g., Krueger, 1978; Proctor, 1982; Ratcliff, 1985), C. W. Eriksen, O'Hara, and B. A. Eriksen (1982) proposed that the longer RT for different judgments was due to response conflict that occurs when feature overlap between different stimuli partially primes same 
responses. In line with this theory, St. James and C. W. Eriksen (in press) have compared same, slightly different, and very different stimulus pairs, and found increases in both RT and DRs with slightly different pairs that had considerable feature overlap, but not for very different pairs. It is not obvious how this would arise from perceptual-level mechanisms.

Although the research reported here involved the use of the response-conflict paradigm of B. A. Eriksen and C. W. Eriksen (1974), and in some respects parallels the work of Coles et al. (1985) and C. W. Eriksen et al. (1985), it does not add to their contributions to the debate concerning "continuous flow" conceptions of information processing (C. W. Eriksen \& Schultz, 1979). As Megaw (1972) has pointed out, however, the mere existence of DRs causes problems for a strictly discrete-stage model of the information processing leading up to the response. DRs themselves may represent either error correction, or the production of a second, stronger response signal "overriding" the first-aborting it and replacing it with a new response. In the latter case, the existence of a second, correct response makes it clear that the system producing the responses cannot be made up wholly of discrete stages, where each stage passes a single output to the next stage only when processing is complete. Such a system simply has no mechanism for generating the second response. Megaw viewed DRs as error corrections, and his argument in this regard is worth reviewing briefly. If DRs are error corrections, then at some point in the system there must be an "error message" of some kind, which must be based on a comparison between the input to a later stage and what that input should have been. An error could arise at either of two placeseither the output from a previous stage contains an error, or the output from that stage is garbled in transmission to the next. In the first case (output error), error correction would require that the earlier stage must have reached a different outcome after the initial signal to the later stage. That is, no error correction could occur if the input to a later stage was compared to the output of an earlier stage unless that output was now different-unless processing had continued after the first signal was sent to the later stage. (Though Megaw did not address the frequency of DRs as a function of stimulus type, DRs would arise more often with incompatible noise because only on those trials are multiple letter identities being activated, so that there is a chance of sending the wrong output initially, with further processing arriving at the correct output.) In the second case (garbled transmission), the error correction could be based on comparing the input to one stage and the output of the previous stage. This mechanism could give rise to DRs even if each stage was discrete and yielded a single output. There is a problem for this interpretation of DRs, however. To preserve a single-discrete-stage model of DRs, some mechanism must be added to explain why errors arise in transmitting the discrete output of one stage to the next. The data presented here produce an additional complication-why do transmission errors arise more frequently with incompatible noise? Unless a discrete-stage model can provide some account of why errors of transmission between stages occur more often for some stimuli than others, a model permitting more than a single output from at least some stages seems more strongly supported.

\section{CONCLUSION}

Measures of emitted responses beyond just the latency to initiate or complete a movement have proven to be informative as to the processes leading to their production (see, e.g., Coles et al., 1985; DeJong, et al., 1990). The evidence presented here, based on such a measure, suggests that the frequency of DRs is both a sensitive and a less technically demanding indicator of the presence of response conflict. Further exploration of the microstructure of responses is likely to be a fruitful enterprise.

\section{REFERENCES}

Coles, M. G. H., Gratton, G., Bashore, T. R., Eruksen, C. W., \& Donchin, E. (1985). A psychophysiological investigation of the continuous flow model of human information processing. Journal of Experimental Psychology: Human Perception \& Performance, 11, 529-553.

DeJong, R., Coles, M. G. H., Logan, G. D., \& Gratton, G. (1990). In search of the point of no return: The control of response processes. Journal of Experimental Psychology: Human Perception \& Performance, 16, 164-182.

Eriksen, B. A., \& Eriksen, C. W. (1974). Effects of noise letters upon the identification of a target letter in a nonsearch task. Perception \& Psychophysics, 16, 143-149.

Eriksen, C. W., Coles, M. G. H., Morrus, L. R., \& O'Hara, W. P. (1985). An electomyographic examination of response competition. Bulletin of the Psychonomic Society, 23, 165-168.

Eriksen, C. W., O'Hara, W. P., \& Eriksen, B. [A]. (1982). Response competition effects in same-different judgments. Perception \& Psychophysics, 32, 261-270.

ERIKSEN, C. W., \& SChultz, D. W. (1979). Information processing in visual search: A continuous flow conception and experimental results. Perception \& Psychophysics, 25, 249-263.

Grabs, C. B. (1965). Probability learning in step-input tracking. British Journal of Psychology, 56, 233-242.

HigGins, J. R., \& ANGEL, R. W. (1970). Correction of tracking errors without sensory feedback. Journal of Experimental Psychology, 84, 412-416.

Kantowitz, B. H. (1973). Response force as an indicant of conflict in double stimulation. Journal of Experimental Psychology, 100, 302-309.

Krueger, L. E. (1978). A theory of perceptual matching. Psychological Review, 85, 278-304.

Megaw, E. D. (1972). Directional errors and their correction in a discrete tracking task. Ergonomics, 15, 633-643.

Proctor, R. W. (1982). A unified theory for matching-task phenomena. Psychological Review, 88, 291-326.

RATCLIFF, R. (1985). Theoretical interpretations of the speed and accuracy of positive and negative responses. Psychological Review, 92 , 212-225.

St. JAmes, J. D., ERIKSEN, C. W. (in press). Response competition produces a "fast-same effect" in same-different judgments. In J. Pomerantz \& G. Lockhead (Eds.), Uncertainty and structure as psychological concepts. Washington, DC: American Psychological Association.

(Manuscript received May 24, 1989; revision accepted for publication July 30,1990 .) 